Acta Crystallographica Section F

Structural Biology

and Crystallization

Communications

ISSN 1744-3091

Simon R. Bushell, ${ }^{a}$ Hubing Lou, ${ }^{a}$

Gregor D. Wallat, ${ }^{\text {a }}$ Konstantinos

Beis, $^{a} \ddagger$ Chris Whitfield ${ }^{b}$ and

James H. Naismith ${ }^{\mathrm{a} *}$

aBiomedical Sciences Research Complex, University of St Andrews, North Haugh, St Andrews KY16 9ST, Scotland, and

${ }^{\mathbf{b}}$ Department of Molecular and Cell Biology, University of Guelph, Ontario N1G 2W1, Canada

₹ Current address: Membrane Protein Laboratory, Diamond Light Source, Imperial College, Chilton OX11 ODE, England.

Correspondence e-mail: naismith@st-andrews.ac.uk

Received 20 September 2010 Accepted 9 October 2010

\section{Crystallization and preliminary diffraction analysis of Wzi, a member of the capsule export and assembly pathway in Escherichia coli}

External polysaccharide capsules provide a physical barrier that is employed by many species of bacteria for the purposes of host evasion and persistence. Wzi is a $53 \mathrm{kDa}$ outer membrane $\beta$-barrel protein that is thought to play a role in the attachment of group 1 capsular polysaccharides to the cell surface. The purification and crystallization of an Escherichia coli homologue of Wzi is reported and diffraction data from native and selenomethionine-incorporated protein crystals are presented. Crystals of C-terminally $\mathrm{His}_{6}$-tagged Wzi diffracted to $2.8 \AA$ resolution. Data processing showed that the crystals belonged to the orthorhombic space group $C 222$, with unit-cell parameters $a=128.8, b=152.8, c=94.4 \AA, \alpha=\beta=\gamma=90^{\circ}$. A His-tagged selenomethioninecontaining variant of Wzi has also been crystallized in the same space group and diffraction data have been recorded to $3.8 \AA$ resolution. Data processing shows that the variant crystal has similar unit-cell parameters to the native crystal.

\section{Introduction}

Extracellular polysaccharide capsules are produced by many species of bacteria to provide protection from the environment. In pathogens, capsules are often considered to be antiphagocytic structures, but they play other diverse roles depending on the species and the structure of the polysaccharide (Horwitz, 1982). The capsule primarily consists of long chains of repeat-unit polysaccharides that are firmly attached to the bacterial outer membrane by processes that are frequently not understood. Structural variation in Escherichia coli capsular polysaccharides (CPSs) gives rise to more than 80 different $\mathrm{K}$ (capsular) antigen types (Whitfield, 2006). Capsules are classified into four groups depending on various biochemical and genetic criteria (Whitfield, 2006; Whitfield \& Roberts, 1999). The model system for group 1 capsules is the $E$. coli $\mathrm{K} 30$ serotype, which utilizes the Wzy-dependent pathway for capsular synthesis and export (Fig. 1).

In E. coli $\mathrm{K} 30$, the $16 \mathrm{~kb}$ capsule-biosynthesis (cps) gene cluster encodes activities required for the biosynthesis, export and assembly of the capsular structure. The locus contains $12 \mathrm{ORFs}$, four of which $(w z i, w z a, w z c$ and $w z b)$ encode proteins involved in the assembly and export of CPS, while the remainder encode proteins responsible for CPS biogenesis. Undecaprenol diphosphate-linked CPS repeat units are synthesized at the cytoplasmic face of the inner membrane and are transported across the bilayer by the flippase Wzx. In the periplasm, these building blocks are polymerized by Wzy (Whitfield, 2006). Higher order polymerization is facilitated by Wzc, a transmembrane tyrosine kinase whose function is moderated by Wzb, its cytoplasmic cognate phosphatase (Hagelueken et al., 2009; Wugeditsch et al., 2001). The completed polymer is then transported to the bacterial surface via Wza, which acts as the pathway's outer membrane channel (Beis et al., 2004; Dong et al., 2006; Nesper et al., 2003).

Wzi is not essential for CPS synthesis (Drummelsmith \& Whitfield, 1999); however, wzi knockouts in both E. coli and Klebsiella spp. 
(which use virtually identical Wzi, Wza, Wzb and Wzc proteins; Rahn et al., 1999) show a profoundly altered morphology of the capsule (Alvarez et al., 2000; Rahn et al., 2003). This is the result of a markedly increased secretion of unattached exopolysaccharide into the environment and a commensurate decrease in attached CPS (Alvarez et al., 2000; Rahn et al., 2003). Wzi proteins are found in representatives from pathogens including E. coli and the genera Acinetobacter, Klebsiella, Providencia and Serratia, as well as freeliving bacteria such as Shewanella. Interestingly, several other bacteria utilize similar Wzy-dependent pathways for the biosynthesis of predominantly secreted, rather than attached, exopolysaccharides. Although these bacteria contain homologues of Wza, Wzb and Wzc (which are presumably needed to make and export polysaccharide), they lack any obvious homologue of Wzi. Examples of these exopolysaccharides include amylovoran and stewartan from the plant pathogens Erwinia amylovora (Bugert \& Geider, 1995) and E. stewartii (Carlier et al., 2009), respectively, and colanic acid from Escherichia coli (Stevenson et al., 1996). Taken together, the collective evidence strongly suggests that Wzi plays a key role in the attachment of the capsule to the bacterial surface. By determining its structure, it may be possible to determine how it performs this vital role. In this report, we describe the purification and crystallization of recombinant Wzi and report on the progress of crystallographic diffraction studies.

\section{Experimental}

\subsection{Expression and purification}

DNA encoding the Wzi homologue from E. coli $\mathrm{B} 44$ (O9:K30: $\left.\mathrm{H}^{-}\right)$ was amplified using PCR primers that introduced a C-terminal hexahistidine tag into the gene product and was cloned into a pBAD24 vector, forming the plasmid pWQ192, as described previously (Rahn et al., 2003). N-terminal tagging was inappropriate as Wzi has a signal sequence that is cleaved during passage across the inner membrane. For the expression of native $\mathrm{Wzi}^{-\mathrm{His}_{6}}$, overnight cultures of E. coli DH5 $\alpha$ (transformed to ampicillin resistance with pWQ192) were grown at $310 \mathrm{~K}$ in Luria-Bertani (LB) medium supplemented with ampicillin $\left(100 \mu \mathrm{g} \mathrm{ml}^{-1}\right)$ and used to inoculate $101 \mathrm{LB}$ broth. Expression of Wzi was induced via the addition of $0.02 \%(w / v)$ arabinose upon the cells reaching an $\mathrm{OD}_{600}$ of $\sim 0.6$. Expression continued for $4 \mathrm{~h}$ at $310 \mathrm{~K}$ before the cells were harvested by centrifugation and stored at $193 \mathrm{~K}$ until required.

For the expression of selenomethionine-variant Wzi-His $_{6}$, the methionine auxotroph E. coli B834 (DE3) strain was transformed to ampicillin resistance with pWQ192. Cultures of the transformed bacteria were grown overnight in LB in the presence of $100 \mu \mathrm{g} \mathrm{ml}^{-1}$ ampicillin. Overnight cultures were harvested by centrifugation ( $4250 \mathrm{~g}$ for $20 \mathrm{~min}$ at $293 \mathrm{~K}$ ) and washed twice with PBS before being used to inoculate $6 \times 11$ Glucose-Free SelenoMet Medium (Molecular Dimensions, Newmarket, England) supplemented with $0.4 \%(v / v)$ glycerol as a carbon source and $100 \mu \mathrm{g} \mathrm{ml}^{-1}$ ampicillin. The media contained $40 \mu \mathrm{g} \mathrm{ml}^{-1} \mathrm{~L}(+)$-selenomethionine and were prepared according to the manufacturer's instructions. Cells were grown at $310 \mathrm{~K}$ to an $\mathrm{OD}_{600}$ of $\sim 0.6$ and expression was induced by the addition of $0.02 \%(w / v)$ arabinose for $4 \mathrm{~h}$ at $310 \mathrm{~K}$. Expression was continued overnight at $298 \mathrm{~K}$ before the cells were harvested by centrifugation at $7000 \mathrm{~g}$ and frozen at $193 \mathrm{~K}$ until required.

Both native and selenomethionine-labelled recombinant Wzi-His $_{6}$ were purified from harvested cells using a modified protocol adapted from Rahn et al. (2003). Frozen cells were thawed in buffer $A$ ( $20 \mathrm{mM}$ phosphate buffer $\mathrm{pH} 7.4,50 \mathrm{mM} \mathrm{NaCl}$ ) and mechanically lysed using a pre-chilled Constant Systems Z Plus series cell disrupter (Constant Systems, Northants, England). Unbroken cells and cell debris were removed by centrifugation at $6000 \mathrm{~g}$ for $20 \mathrm{~min}$. The membrane fraction was isolated from the resultant supernatant by ultracentrifugation at $120000 \mathrm{~g}$ for $1 \mathrm{~h}$. Inner membranes in the total membrane fraction were selectively solubilized by resuspending the pellet in buffer $A$ containing 2\%(w/v) $N$-lauroylsarcosine (SigmaAldrich UK, catalogue No. L5125) and incubating at $277 \mathrm{~K}$ with stirring for $3 \mathrm{~h}$. The volume of this solubilization was $20 \mathrm{ml}$ per litre of original culture. The outer membrane fraction was isolated as a pellet

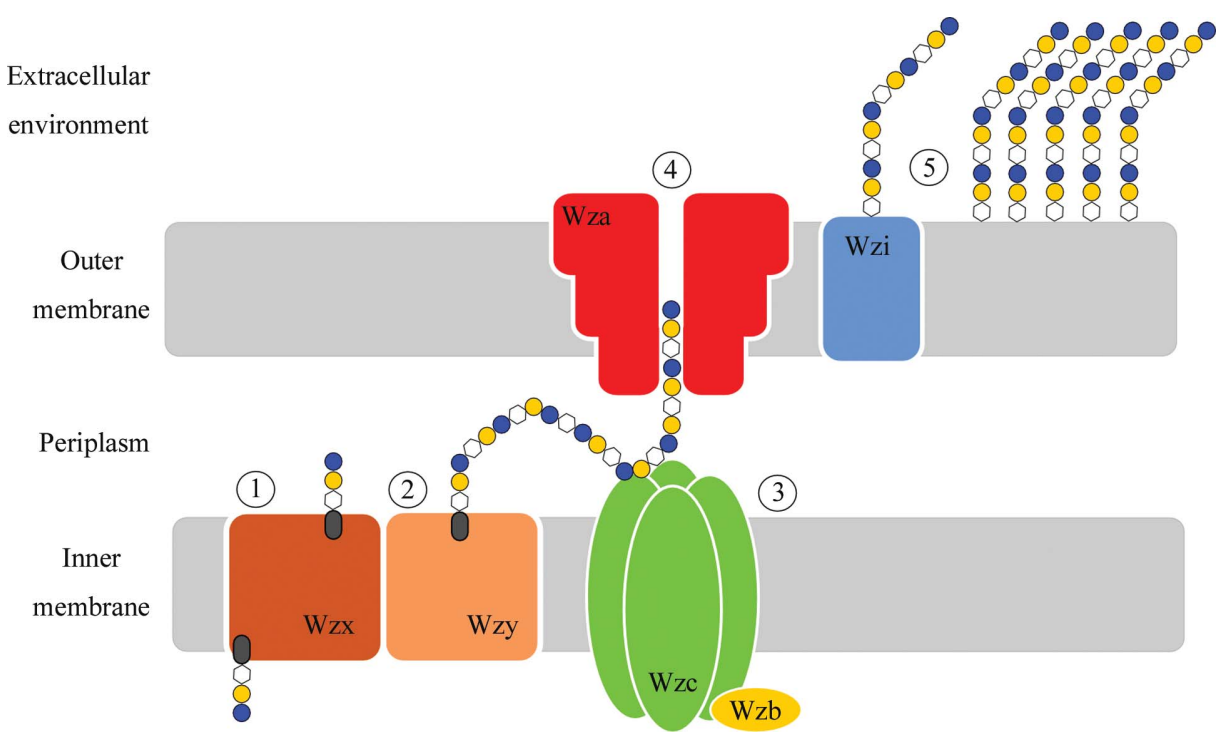

Figure 1

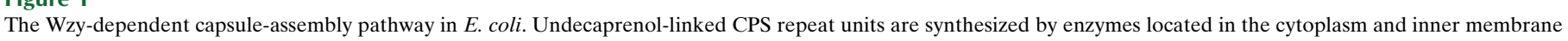

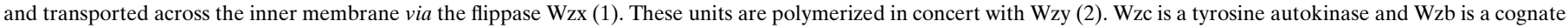

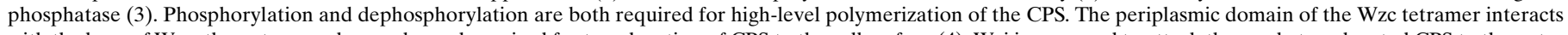

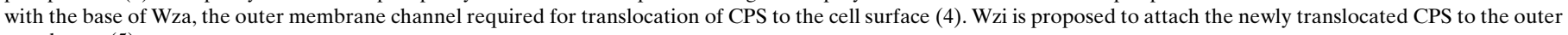
membrane (5). 
after further ultracentrifugation at $120000 \mathrm{~g}$ for $1 \mathrm{~h}$ and solubilized overnight in $120 \mathrm{ml}$ buffer $A$ containing $0.5 \%(w / v)$ SB3.14 [3- $(N, N-$ dimethylmyristylammonio)propanesulfonate; Sigma-Aldrich UK,

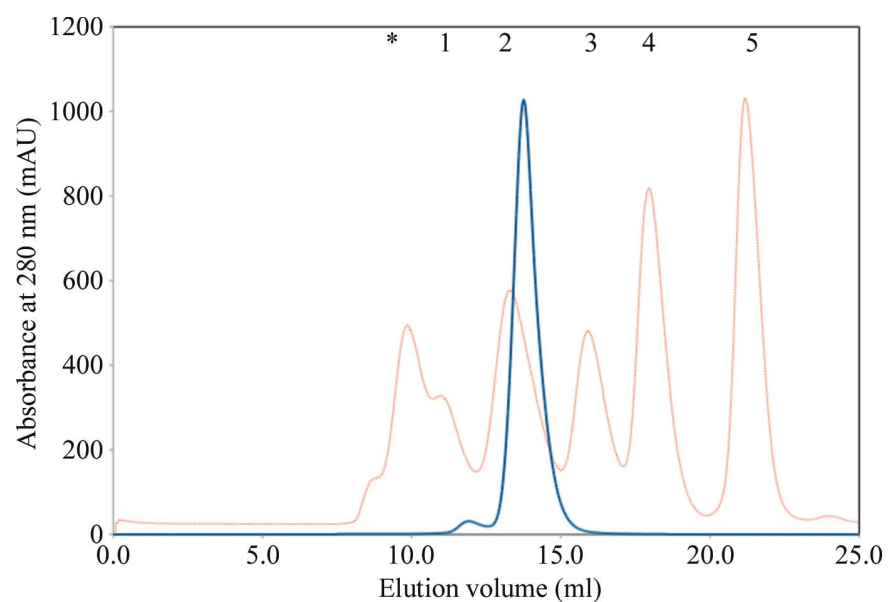

(a)

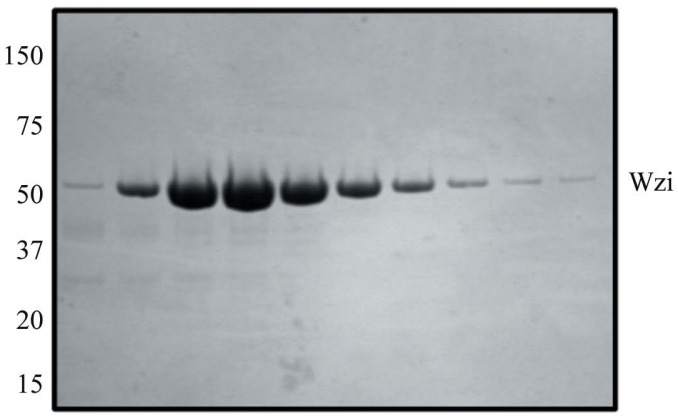

(b)

Figure 2

Purification of native $\mathrm{Wzi}^{-\mathrm{His}_{6} .}$ ( $a$ ) Elution profile of native $\mathrm{Wzi}^{-\mathrm{His}_{6}}$ (blue line) on size-exclusion chromatography using a Superdex 200 10/300 column. The elution was standardized using a mixture containing (1) thyroglobulin $(670 \mathrm{kDa}),(2)$ $\gamma$-globulin (158 kDa), (3) ovalbumin (44 kDa), (4) myoglobin (17 kDa) and (5) vitamin $\mathrm{B}_{12}(1350 \mathrm{Da})$ (red line). The protein aggregate peak (marked with an asterisk) represents the void volume of the column. (b) SDS-PAGE analysis of peak fractions from SEC purification, showing the homogeneity of Wzi-His 6 immediately prior to crystallization. The protein runs to its true size of $52 \mathrm{kDa}$ upon boiling. catalogue No. T7763]. Insoluble material was removed by ultracentrifugation at $120000 \mathrm{~g}$ for $1 \mathrm{~h}$. The supernatant was applied onto a $5 \mathrm{ml}$ HisTrap nickel column (GE Healthcare, Uppsala, Sweden) preequilibrated in $20 \mathrm{~m} M$ sodium phosphate $\mathrm{pH} 7.4,50 \mathrm{~m} M \mathrm{NaCl}$, $0.05 \%(w / v)$ SB3.14. Once Wzi-His 6 was bound, the column was washed with five column volumes of $20 \mathrm{~m} M$ sodium phosphate $\mathrm{pH}$ 7.4, $50 \mathrm{~m} M \mathrm{NaCl}, 0.1 \%(w / v)$ LDAO ( $n$-dodecyl- $N, N$-dimethylamine$N$-oxide; Anatrace, Maumee, USA, catalogue No. D360). Wzi-His was eluted by a step gradient of wash buffer with increasing amounts of imidazole $(12.5,25,50,100$ and $500 \mathrm{mM})$. Wzi-His 6 readily eluted in $50 \mathrm{~m} M$ imidazole, which is an unusually low concentration for a hexahistidine-tagged protein; however, SDS-PAGE analysis of peak fractions showed it to be nearly homogenous. Final purification was performed via gel-filtration chromatography using a Superdex 200 10/300 size-exclusion column (GE Healthcare, Uppsala, Sweden) equilibrated in $20 \mathrm{~m} M$ Tris $\mathrm{pH} 7.4,0.1 \%$ LDAO. Gel filtration of the selenomethionine form of Wzi-His $_{6}$ used the same buffer but supplemented with $0.5 \mathrm{~m} M$ tris(2-carboxyethyl)phosphine (TCEP).

The elution profile indicated a single oligomeric state of $\mathrm{Wzi}^{-\mathrm{His}_{6}}$ in solution. Comparison with known protein standards suggested that Wzi elutes as a monomer-detergent complex (Fig. 2a). When plotted on a calibration curve, the Wzi-detergent complex was calculated to have an approximate molecular weight of $80 \mathrm{kDa}$, which is in reasonable agreement with the molecular weight of Wzi of $53 \mathrm{kDa}$ and an LDAO micelle size of $\sim 25 \mathrm{kDa}$ (Strop \& Brunger, 2005). The purification purity was monitored at all stages via SDS-PAGE (Fig. 2b), with no other contaminants being visible on a Coomassie Blue-stained gel. The identity of recombinant $\mathrm{Wzi}^{-H_{i s}}{ }_{6}$ was confirmed by Western blot using an antihexahistidine monoclonal antibody and via MALDI-TOF mass-spectrometric analysis of peptides derived from a tryptic digest. The final yield of the native form of the protein was approximately $0.4 \mathrm{mg}$ per litre of culture. The yield of the selenomethionine variant was higher at $0.7 \mathrm{mg}$ per litre of culture, most likely owing to the longer overnight expression. Wzi was concentrated to $\sim 12 \mathrm{mg} \mathrm{ml}^{-1}$ for crystallization trials.

\subsection{Crystallization}

Several commercially available screens were employed to determine the crystallization space of Wzi-His $_{6}$. Crystallization trays were generally constructed using a Cartesian Honeybee 963 liquid-handing

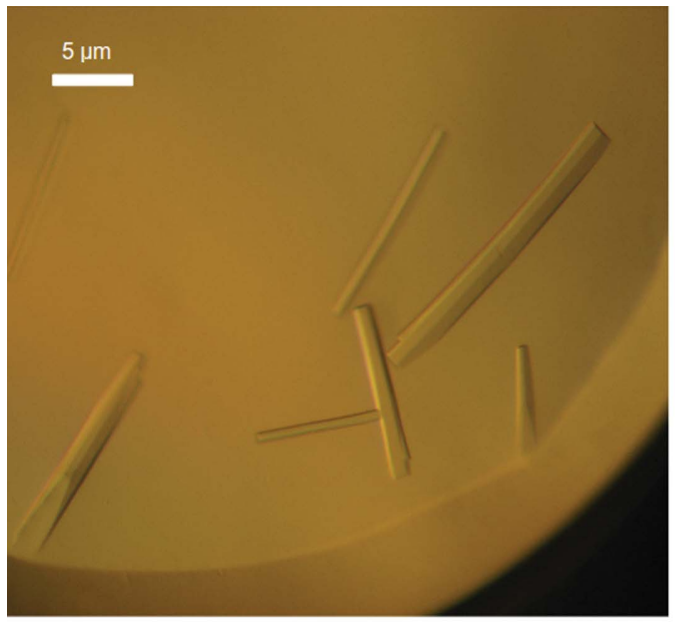

(a)

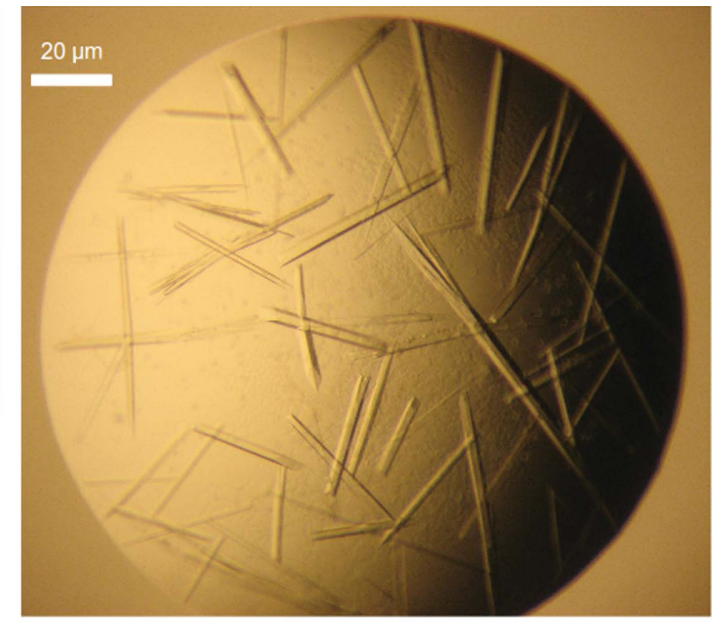

(b)

Figure 3

Crystals of native and selenomethionine-containing forms of Wzi-His $6 .(a)$ Native crystals grown in $0.03 M \mathrm{CaCl}_{2}, 25 \%(v / v)$ PEG $350 \mathrm{MME}$, 0.1 $M$ MES pH 6.5. (b)

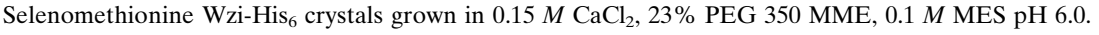


robot in Innovadyne SD-2 96-well trays (IDEX Corp, Lake Forest, USA) with $60 \mu \mathrm{l}$ precipitant dispensed into the reservoir. Crystallization was performed via sitting-drop vapour diffusion at $293 \mathrm{~K}$ by mixing $150 \mathrm{nl}$ Wzi-His $_{6}$ solution (at $12 \mathrm{mg} \mathrm{ml}^{-1}$ ) with $150 \mathrm{nl}$ well solution. Native Wzi-His ${ }_{6}$ crystallized in several different conditions, with crystals taking between one and eight weeks to appear. Ovalshaped crystals grew in $12.5 \%(w / v)$ PEG $200 \mathrm{MME}, 0.1 \mathrm{M}$ sodium cacodylate $\mathrm{pH} 6.5$; however, these crystals proved to be difficult to reproduce and were marked by severe anisotropy, most likely owing

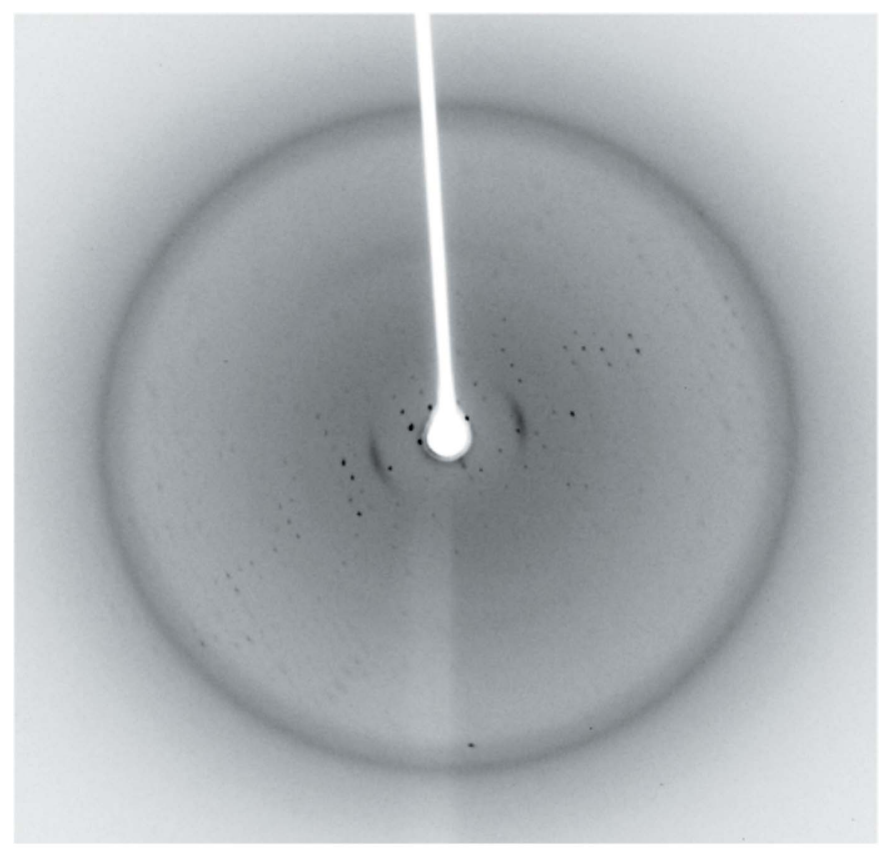

(a)

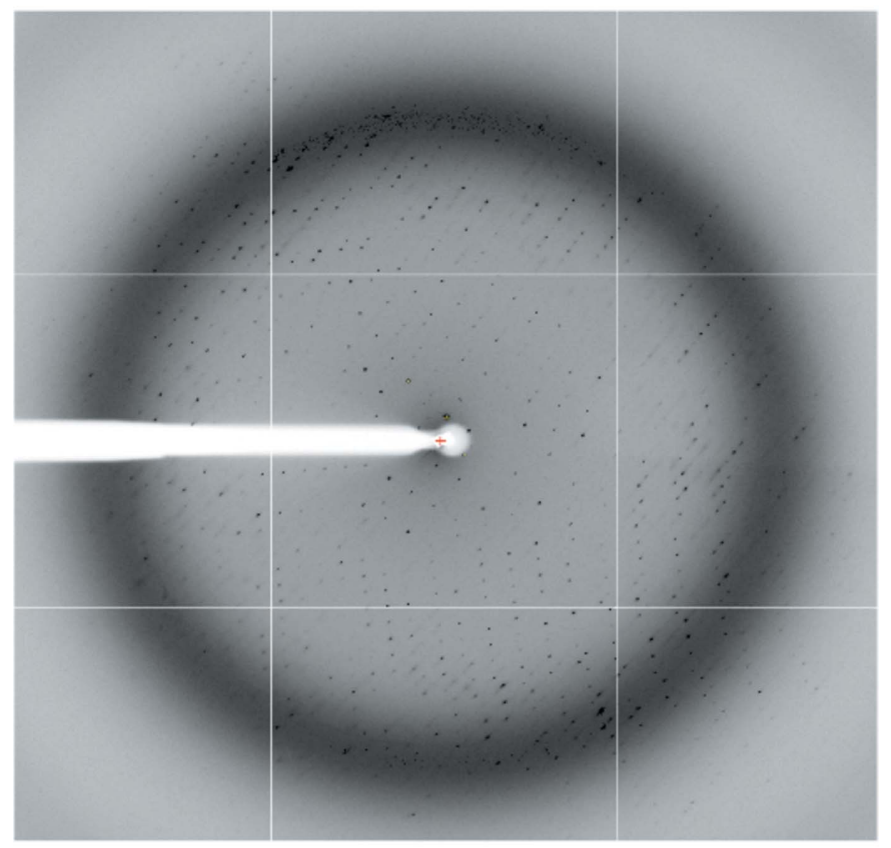

(b)

Figure 4

Native crystal diffraction. (a) Diffraction pattern from a typical native Wzi-His ${ }_{6}$ crystal showing high anisotropy. Data were collected in-house using a Saturn 944+ CCD detector attached to a Rigaku MicroMax-007 HFM X-ray source $(\lambda=1.54178 \AA$ ). Such crystals were discarded without further experimentation. (b) Diffraction pattern from the native $\mathrm{Wzi}^{-} \mathrm{His}_{6}$ crystal used to derive the data in Table 1 .
Table 1

Data-collection and processing statistics.

Values in parentheses are for the last shell.

\begin{tabular}{lll}
\hline & Native Wzi & SeMet Wzi \\
\hline Source & ESRF, ID14-4 & DLS, I24 \\
Wavelength $(\AA)$ & 0.9255 & 0.9778 \\
Oscillation $\left(^{\circ}\right)$ & 0.65 & 1.0 \\
Space group & $C 222$ & $C 222$ \\
Unit-cell parameters & $a=133.2, b=152.3, c=95.3$, & $a=128.8, b=152.8, c=94.4$, \\
$\quad\left(\AA{ }^{\circ}\right)$ & $\alpha=\beta=\gamma=90$ & $\alpha=\beta=\gamma=90$ \\
Resolution $(\AA)$ & $50-2.8$ & $53-3.8$ \\
Total reflections & 132346 & 41664 \\
Unique reflections & 23861 & 9484 \\
Mosaicity $\left({ }^{\circ}\right)$ & 0.72 & 0.14 \\
Anomalous correlation & - & $0.114(0.047)$ \\
Completeness $(\%)$ & $98.5(98.7)$ & $99.9(100)$ \\
Multiplicity & $5.5(4.7)$ & $4.4(4.6)$ \\
Mean $I / \sigma(I)$ & $13.7(2.7)$ & $7.6(3.0)$ \\
$R_{\text {merge }}(\%)$ & $8.2(52.2)$ & $14.5(40.3)$ \\
\hline
\end{tabular}

to their shape. The best crystals appeared in screens optimized for the crystallization of membrane proteins, specifically MembFac (Hampton Research, Aliso Vijeo, USA), MemGold and MemStart/ MemSys (Molecular Dimensions, Newmarket, England). Broad screen hits were improved by varying the $\mathrm{pH}$, salt and PEG concentrations. The most tractable crystals grew in $0.03 M \mathrm{CaCl}_{2}$, $25 \%(v / v)$ PEG 350 MME, $0.1 M$ MES pH 6.5. The crystals resembled flattened hexagonal rods and usually grew attached to the bottom of the crystallization well. Small crystals appeared after $\sim 3 \mathrm{~d}$ and grew to approximately $30 \mu \mathrm{m}$ in size within $7-10 \mathrm{~d}$ (Fig. $3 a$ ).

Selenomethionine-labelled $\mathrm{Wzi}^{-\mathrm{His}_{6}}$ crystals grew under slightly different conditions, with the best crystals forming in $0.15 \mathrm{M} \mathrm{CaCl}_{2}$, 23\% PEG $350 \mathrm{MME}, 0.1 \mathrm{M}$ MES pH 6.0. These crystals were of a similar morphology to those of the native form and grew to a comparable size (Fig. 3b). However, these crystals grew more quickly, appearing overnight and growing to full size $(30 \mu \mathrm{m})$ within $2-3 \mathrm{~d}$.

All crystals used the drop itself as a cryoprotectant. Crystals were flash-cooled in liquid $\mathrm{N}_{2}$, either through immersion in pre-cooled robot pucks or by freezing the crystals directly in the cryostream prior to collection. Initial diffraction data showed that the majority of crystals frequently displayed moderate to severe anisotropy. Variation of cryoprotection conditions did not demonstrate any improvement of this problem. Rather, crystals were screened in advance in order to select the least anisotropic for further data collection.

\subsection{Data collection}

Data sets from native Wzi-His $_{6}$ crystals were collected on the ID14-4 beamline at the ESRF, Grenoble, France. A $2.8 \AA$ resolution data set (Fig. 4) was collected from a single native Wzi-His $_{6}$ crystal. The data were processed with $X D S$ using the xia2 data-reduction pipeline (Kabsch, 2010; Winter, 2010). xia2 used POINTLESS and $L A B E L I T$ to assist it in indexing the data (Collaborative Computational Project, Number 4, 1994; Sauter et al., 2004). During processing, several wedges of data were reduced separately in xia 2 and subsequently merged with SCALA (Evans, 2006).

Data processing showed that the native crystals belonged to the orthorhombic space group $C 222$, with unit-cell parameters $a=128.8$, $b=152.8, c=94.4 \AA, \alpha=\beta=\gamma=90^{\circ}$. The full details of this data set are described in Table 1. Matthews coefficient analysis suggested that either one or two monomers of the protein are present in the asymmetric unit ( $V_{\mathrm{M}}=4.46$ or $2.23 \AA^{3} \mathrm{Da}^{-1}$, respectively), with a solvent content of 72 or $45 \%$, respectively (Kantardjieff \& Rupp, 2003; Matthews, 1968). Although $72 \%$ solvent content is at the upper range of commonly observed values, the anisotropic nature of the 
diffraction, the absence of any noncrystallographic symmetry and the fragility of the crystals point towards the presence of a monomer in the asymmetric unit.

Although the sequence of Wzi suggests that it is a $\beta$-barrel, we have not identified a matching structure and all attempts at molecular replacement failed. The selenomethionine variant of $\mathrm{Wzi}^{-\mathrm{His}_{6}}$ was crystallized in order to determine the phases using single-wavelength anomalous dispersion (SAD). Data sets from crystals that showed promising in-house diffraction were collected on beamline I24 at the Diamond Light Source (DLS), Didcot, England using a Pilatus 6M detector $(\lambda=0.9778 \AA)$. Several data sets were collected, with the best example containing diffaction to $3.8 \AA$ resolution. These data were indexed and scaled using XDS in xia 2 using the same approach as described for the native form (Kabsch, 2010; Winter, 2010). Processing showed that these crystals also belonged to space group C222 and they showed similar unit-cell parameters to the native protein (Table 1). Fluorescence scans of crystals of the selenomethionine variant showed that selenomethionine had been incorporated into the protein. Data reduction indicated the presence of anomalous scattering by measuring the difference between $\left|I^{-}\right|$and $\left|I^{+}\right|$. We have not yet been able to solve the substructure of Se atoms.

\section{Discussion}

We have obtained crystals of the integral membrane E. coli protein Wzi. Although crystals appeared in initial screens, extensive optimization was required in order to obtain crystals which diffracted to beyond $6 \AA$ resolution. The native crystals diffracted to $2.8 \AA$ resolution, suggesting that it will be possible to report a well defined experimental structure for this protein. Sequence analysis suggests the protein will be a $\beta$-barrel, but with a structure different to those observed previously. Gel filtration and crystal packing are consistent with a monomeric protein. We anticipate that solution of the structure of the selenomethionine variant of $\mathrm{Wzi}^{-\mathrm{His}_{6}}$ will require higher quality diffraction data. Efforts to improve the crystal quality for the selenomethionine protein are continuing.
This work was supported by funding from the Wellcome Trust (program grant to JHN and CW). Access to the Diamond and ESRF synchrotrons is gratefully acknowledged. The authors would also like to gratefully acknowledge Ms Victoria Elder (St Andrews) for her valuable input.

\section{References}

Alvarez, D., Merino, S., Tomás, J. M., Benedí, V. J. \& Albertí, S. (2000). Infect. Immun. 68, 953-955.

Beis, K., Collins, R. F., Ford, R. C., Kamis, A. B., Whitfield, C. \& Naismith, J. H. (2004). J. Biol. Chem. 279, 28227-28232.

Bugert, P. \& Geider, K. (1995). Mol. Microbiol. 15, 917-933.

Carlier, A., Burbank, L. \& von Bodman, S. B. (2009). Mol. Microbiol. 74, 903-913.

Collaborative Computational Project, Number 4 (1994). Acta Cryst. D50, 760-763.

Dong, C., Beis, K., Nesper, J., Brunkan-Lamontagne, A. L., Clarke, B. R., Whitfield, C. \& Naismith, J. H. (2006). Nature (London), 444, 226229.

Drummelsmith, J. \& Whitfield, C. (1999). Mol. Microbiol. 31, 1321-1332.

Evans, P. (2006). Acta Cryst. D62, 72-82.

Hagelueken, G., Huang, H., Mainprize, I. L., Whitfield, C. \& Naismith, J. H. (2009). J. Mol. Biol. 392, 678-688.

Horwitz, M. A. (1982). Rev. Infect. Dis. 4, 104-123.

Kabsch, W. (2010). Acta Cryst. D66, 125-132.

Kantardjieff, K. A. \& Rupp, B. (2003). Protein Sci. 12, 1865-1871.

Matthews, B. W. (1968). J. Mol. Biol. 33, 491-497.

Nesper, J., Hill, C. M. D., Paiment, A., Harauz, G., Beis, K., Naismith, J. H. \& Whitfield, C. (2003). J. Biol. Chem. 278, 49763-49772.

Rahn, A., Beis, K., Naismith, J. H. \& Whitfield, C. (2003). J. Bacteriol. 185, 5882-5890.

Rahn, A., Drummelsmith, J. \& Whitfield, C. (1999). J. Bacteriol. 181, 23072313.

Sauter, N. K., Grosse-Kunstleve, R. W. \& Adams, P. D. (2004). J. Appl. Cryst. 37, 399-409.

Stevenson, G., Andrianopoulos, K., Hobbs, M. \& Reeves, P. R. (1996). J. Bacteriol. 178, 4885-4893.

Strop, P. \& Brunger, A. T. (2005). Protein Sci. 14, 2207-2211.

Whitfield, C. (2006). Annu. Rev. Biochem. 75, 39-68.

Whitfield, C. \& Roberts, I. S. (1999). Mol. Microbiol. 31, 1307-1319.

Winter, G. (2010). J. Appl. Cryst. 43, 186-190.

Wugeditsch, T., Paiment, A., Hocking, J., Drummelsmith, J., Forrester, C. \& Whitfield, C. (2001). J. Biol. Chem. 276, 2361-2371. 